\title{
Modeling the viscoelasto-plastic behavior of waxy crude
}

\section{Teng Houxing and Zhang Jinjun*}

\author{
National Engineering Laboratory for Pipeline Safety/Beijing Key Laboratory of Oil and Gas Distribution Technology, China \\ University of Petroleum, Beijing 102249, China
}

(C) China University of Petroleum (Beijing) and Springer-Verlag Berlin Heidelberg 2013

\begin{abstract}
Waxy crude oil exhibits complex rheological behavior below the pour point temperature, such as viscoelasticity, yield stress, and thixotropy, owing to the formation of a three-dimensional spongelike interlock network structure. This viscoelasto-thixotropic behavior is an important rheological behavior of waxy crude oils, determining the flow recovery and safe restart of crude oil pipelines. Up to now, the thixotropic models for waxy crude have been all viscoplastic models, without considering the viscoelastic part before the yield point. In this work, based on analyzing the variation of the elastic stress and viscous stress in the Mujumbar model, a new viscoelasto-plastic model is proposed, whose shear stress is separated into an elastic component and a viscous component. The elastic stress is the product of the shear modulus and elastic strain; the shear modulus is proportional to the structural parameter. For the elastic strain, we followed the line of Zhu and his coauthors and assumed that it may be expressed by an algebraic equation. The model is validated by stepwise shear rate tests and hysteresis loop tests on Daqing and Zhongyuan waxy crude. The results show that the model's fitting and predictive capability is satisfactory.
\end{abstract}

Key words: Thixotropy, viscoelasticity, viscoelasto-plastic model, thixotropic model, waxy crude

\section{Introduction}

More than $80 \%$ of crude oil produced in China is waxy oil, such as Zhongyuan and Daqing crude oils, with a wax content of $21.5 \%$ and $26.3 \%$, respectively. In the past ten years, the output of waxy crude throughout the world has increased significantly. Below the wax appearance temperature (WAT), wax molecules begin to precipitate and form solid wax crystals in crude oil (Rønningsen, 1992). Below the pour point temperature the precipitated wax crystals interlock with each other to form a three-dimensional spongelike network structure, completing the transition of crude oil from sol to colloidal gel (Visintin et al, 2005; $\mathrm{Zhu}$ et al, 2007). Consequently the gelled waxy crude oil shows complex rheological behavior, such as viscoelasticity, yield stress, and thixotropy (Zhang and Liu, 2008; Magda et al, 2009). Experimental results show that the mechanical response of a gelled waxy crude is linear viscoelastic at the beginning of deformation (Chang et al, 1998). As the shear strain increases, the three dimensional network composed of loose clusters is disrupted into a small number of relatively large flocs or aggregates, losing the connectivity of the network structure (Chang et al, 1998; Visintin et al, 2005). In this process, the viscoelasticity decays, and the mechanical

*Corresponding author. email: zhangjj@cup.edu.cn

Received January 4, 2013 response gradually transfers from being dominated by the elastic effect to being dominated by the viscous effect. After shearing under some high shear rate for a period of time, the internal network structure is nearly totally destroyed, thus the viscoelastic behavior is very weak and even can be ignored, then the rheological response is mainly thixotropic (Chang et al, 1998).

However, most of the existing thixotropic models for waxy crude are viscoplastic models, utilizing a yield stress to characterize the yielding behavior (Barnes, 1997; Mujumdar et al, 2002; Zhang et al, 2010). The viscoplastic models assume that the material begins to flow only if the shear stress is greater than the yield stress. For this reason, they can only depict the thixotropic behavior after the yield point and cannot account for the initial viscoelastic effect before the yield point (Labanda and Llorens, 2006). In recent years, some scholars attempted to describe both the viscoelastic and thixotropic effects of thixotropic materials with yield stress (Barnes, 1997; Mujumdar et al, 2002; Dullaert and Mewis, 2006; de Souza Mendes, 2009; 2011). To characterize the viscoelastic and thixotropic properties of blood, Fang and Jiang (1998) developed a constitutive equation based on the principle of a mechanical analogy composed of a spring, a dashpot, and a self-developed thixotropic element in parallel. However, the elastic stress of the model increases with the shear strain unbounded in time. To overcome this problem, a modified model was developed (Fang et al, 1999). However, 
the elastic stress in the modified model increases from 0 to its maximum value, and then does not drop in the following shearing process, which is not consistent with the thixotropic behavior of waxy crude.

Mujumdar et al (2002) proposed a nonlinear rheological model for thixotropic fluids with yield stress to account for the time-dependent elastic, viscous, and yielding phenomena. The total shear stress is separated into elastic and viscous components which originate from intra-floc deformation and inter-floc dissipation, respectively. And the relative importance of the elastic stress to viscous stress is determined by the structural parameter. Besides, to control the transformation from viscoelastic behavior to yielding behavior, an upper limit of elastic strain is adopted and its evolution with the floc size is expressed by an algebraic relation. Following the line of the Mujumdar model, Huang and $\mathrm{Lu}$ (2005) developed a thixotropic model to describe the time-dependent nonlinear viscoelastic behavior of LDPE melt. The elastic stresses at different shear strains are depicted by a nonlinear damping function $h(\gamma)$ along with a structural parameter $\lambda$, that is $G(\lambda, \gamma)=\lambda G_{0} h(\gamma)$. However, the model does not introduce the concept of elastic strain, bringing about the drawback that the damping function will decrease in the case of the internal structure building up. As to the Mujumdar model and the Huang model, the viscous stress under constant shear rate increases with a decrease in the structural parameter. While for waxy crude and other thixotropic materials with yield stress, after shearing at a high shear rate for a period of time, the internal structure is nearly totally damaged and the elastic stress will be very small, then the total shear stress is mainly composed of the viscous stress. In this condition, the total shear stress predicted by the two models will increase with time, which conflicts with the experimental evidence.

Dullaert and Mewis (2006) developed a general structural kinetics thixotropic model for thixotropic systems based on inelastic suspending fluid, whose shear stress is divided into a particle and a medium contribution, and the particle contribution is further subdivided into an elastic and a viscous hydrodynamic contribution. As for the elastic contribution, a limit or critical elastic strain is also adopted and the evolution of elastic strain is described by a differential kinetic equation. Zhu and Smay (2011) proposed an engineering model to describe the thixotropic rheology of alumina concentrated colloidal gel, and the elastic strain is assumed to be expressed as a simple algebraic equation. At the very beginning of deformation, the shear stress predicted by the two models (Dullaert and Mewis, 2006; Zhu and Smay, 2011) has elastic and viscous components; while for the two models (Mujumdar et al, 2002; Huang and Lu, 2005), the shear stress only has the elastic component without the viscous component. This is one distinction between them. Nevertheless, many papers showed that for waxy crude (Visintin et al, 2005; Oh and Deo, 2008; Oh et al, 2009; Magda et al, 2009) and other thixotropic materials with yield stress (Liddel and Boger, 1996; Putz and Burghelea, 2009), the initial mechanical response is elastic. From this aspect, the initial mechanical response predicted by the Mujumdar and Huang model seems more realistic and plausible for these materials.
The existing viscoelasto-plastic models, developed for other materials whose thixotropic properties differ from waxy crude, fail to capture the viscoelasto-plastic behavior of waxy crude. Therefore, there is an urgent need to establish a rheological model that can fully describe the viscoelastothixotropic behavior of gelled waxy crudes (Livescu, 2012). In this work, we proposed a viscoelasto-plastic model based on the Mujumbar model's analysis on the variation of the elastic and viscous components. As to the evolution of the elastic strain in the model, it is expressed by an algebraic equation, following the line of the model proposed by Zhu and Smay (2011). The proposed model is validated by stepwise shear rate tests and hysteresis loop tests of Daqing and Zhongyuan waxy crude. Moreover, the validity and applicability of the model is further checked by predicting the transient flow curve of the hysteresis loop test with the model parameters determined from the stepwise shear rate test.

\section{Proposed model}

Just like the common practice for the structural kinetics models (Mewis and Wagner, 2009), the structuring level of the microstructure of a material is represented by a nonnegative scaled structural parameter $\lambda$. It varies between the values of 0 for the completely broken-down structure and 1 for the fully developed structure. The proposed model is composed of an equation of state and a kinetic equation for the structural parameter.

\subsection{Equation of state}

As the model proposed by Mujumdar et al (2002), the total shear stress $\tau$ is also composed of an elastic stress $\tau_{\mathrm{e}}$ and a viscous stress $\tau_{\mathrm{v}}$, that is $\tau=\tau_{\mathrm{e}}+\tau_{\mathrm{v}}$. The elastic stress $\tau_{\mathrm{e}}$ is assumed to obey the Hookean elastic response, that is $\tau_{e}=$ $G(\lambda) \gamma_{\mathrm{e}}$, where $G(\lambda)$ is the structure-dependent shear modulus and $\gamma_{\mathrm{e}}$ is the elastic strain caused by the presence of the deformable network structure. When the network structure is complete $(\lambda=1)$, the mechanical response is linear elastic, without a viscous response. With an increase in the total shear strain $\gamma$, the network structure is disrupted into smaller structures or aggregates, resulting in the decay of the shear modulus $G$ and the rise of the viscous behavior. Most of the existing models (Yziquel et al, 1999; Mujumdar et al, 2002; Dullaert and Mewis, 2006; Zhu and Smay, 2011) assume that the shear modulus $G$ is dependent only on the structural parameter $\lambda$. Here, we also assume that the shear modulus $G$ is proportional to instantaneous value of $\lambda$, i.e. $G(\lambda)=\lambda G_{0}$, where $G_{0}$ is the shear modulus of the completely structured material $(\lambda=1)$.

As to the elastic strain $\gamma_{\mathrm{e}}$, the model proposed by Doraiswamy et al (1991) assumes that $\gamma_{\mathrm{e}}$ will increase linearly from zero to some maximum value $\gamma_{c}$ equal to the critical strain at the yield point, and it will remain constant as long as the deformation process continues in the same direction. For the two models (Mujumdar et al, 2002; Dullaert and Mewis, 2006), prior to yielding $\gamma_{\mathrm{c}}$ is the same as the elastic strain in the Doraiswamy model; after yielding the Mujumdar model's critical strain $\gamma_{\mathrm{c}}$ is assumed to be a function of the floc size and expressed as $\gamma_{\mathrm{c}}=\gamma_{\mathrm{co}} \lambda^{m}$, while for the Dullaert 
model, the evolution of $\gamma_{\mathrm{e}}$ with shearing conditions and time is described by a specially proposed differential equation. The elastic strain $\gamma_{\mathrm{e}}$ in the Doraiswamy and Mujumdar model is continuous, but has an inflection point at the yield point. Consequently, the elastic stress $\tau_{\mathrm{e}}$ is also continuous but has an inflection point at the yield point. Theoretically, $\gamma_{\mathrm{e}}$ and $\tau_{\mathrm{e}}$ should be continuous and smooth. As for the model proposed by Zhu and Smay (2011), the elastic strain's assumption is the same as that of the Doraiswamy model, but to overcome the problem of exiting an inflection point at the yield point, $\gamma_{\mathrm{e}}$ is assumed to be expressed by a simple algebraic equation as $\gamma_{\mathrm{e}}=\gamma_{\mathrm{c}}\left[1-\exp \left(-p \dot{\gamma} t^{q}\right)\right]$, where $p$ and $q$ are characteristic parameters. Nevertheless, if the shear rate changes stepwise, the value of $\dot{\gamma} t^{q}$ also changes stepwise, and so do the values of $\gamma_{\mathrm{e}}$ and elastic stress $\tau_{\mathrm{e}}$. To overcome this problem, here we assume that the evolution of $\gamma_{\mathrm{e}}$ may be expressed by $\gamma_{\mathrm{e}}=\gamma_{\mathrm{c}}\left\lfloor 1-\exp \left(-p \gamma^{q}\right)\right\rfloor$, where $\gamma$ is the total shear strain and it has $\gamma=\int_{0}^{t} \dot{\gamma}\left(t^{\prime}\right) \mathrm{d} t^{\prime}$. Then $\tau_{\mathrm{e}}$ can be expressed as $\tau_{\mathrm{e}}=\lambda G_{0} \gamma_{\mathrm{c}}\left[1-\exp \left(-p \gamma^{q}\right)\right]$, where $\gamma_{\mathrm{c}}$ is the shear strain at the yield point. $G_{0} \gamma_{\mathrm{c}}$ is the maximum value of the elastic stress $\tau_{\mathrm{e}}$. For simplicity, here we define $\tau_{y}=G_{0} \gamma_{\mathrm{c}}$ as the maximum of the elastic yield stress. Finally, the elastic stress $\tau_{\mathrm{e}}$ has the following form:

$$
\tau_{\mathrm{e}}=\lambda \tau_{y}\left[1-\exp \left(-p \gamma^{q}\right)\right]
$$

For the viscous stress $\tau_{\mathrm{v}}$, it is assumed as in the model proposed by Houska (1981) that the viscosity is characterized by a structure-dependent consistency $\Delta k$ and a completely unstructured consistency $k$. The dependence of $\tau_{\mathrm{v}}$ on shear rate is described by a kinetic index $n_{1}$. The relative importance of the contributions of $\tau_{\mathrm{e}}$ to $\tau_{\mathrm{v}}$ is determined by the structural parameter $\lambda$ (Mujumdar et al, 2002). Finally, the equation of state takes the form as follows:

$$
\tau=\lambda \tau_{y}\left[1-\exp \left(-p \gamma^{q}\right)\right]+(1-\lambda) \cdot(k+\lambda \Delta k) \dot{\gamma}^{n_{1}}
$$

\subsection{Kinetic equation}

As for the kinetic equation for the structural parameter $\lambda$, in analogy with chemical reaction kinetics, it is usually assumed that its time evolution is controlled by the combined result of structure buildup and breakdown rates. So far many kinetic equations have been put forward, and some of them introduce a common prefactor for the buildup and breakdown terms (Mewis and Wagner, 2009; Jia and Zhang, 2012; Teng and Zhang, 2012). The prefactor does not change the equilibrium value of $\lambda$ at each shear rate. It only changes the descending rate of $\lambda$ coming to its equilibrium value. By this way, it substantially improves the predictive capability of the model. In this work, the prefactor $1 /\left(1+\gamma^{n_{2}}\right)$ with shear strain as a variable, first used in the model (Teng and Zhang, 2012), is introduced. Then the structural parameter $\lambda$ is assumed to obey the following kinetic equation:

$$
\frac{\mathrm{d} \lambda}{\mathrm{d} t}=\frac{1}{1+\gamma^{n_{2}}}[a(1-\lambda)-\lambda f(\tau, \dot{\gamma})]
$$

where $\gamma$ is the total shear strain; $n_{2}$ is a positive dimensionless constant; $a$ is a kinetic constant for structure buildup. In this equation, the first term on the right-hand side represents the structure buildup, while the second term stands for the structure breakdown.

For thixotropic materials with yield stress, it is physically reasonable to expect that the evolution of $\lambda$ has the following characteristics: (i) the breakdown rate is low at early times when the microstructure is nearly un-deformed; (ii) as time elapses and the shear strain increases, the breakdown rate increases; (iii) near the yield point the breakdown rate comes to its maximum value; (iv) after that it begins to decay. Most of the existing kinetic equations assume that the breakdown term $f(\tau, \dot{\gamma})$ is a function of shear rate only (Houska, 1981; Toorman, 1997; Coussot et al, 2002; Mujumdar et al, 2002). If the function $f(\tau, \dot{\gamma})$ is taken to be dependent on shear rate (e.g. $\left.f(\tau, \dot{\gamma}) \propto \dot{\gamma}^{m}\right)$, as analyzed by de Souza Mendes (2009; 2011), $f(\tau, \dot{\gamma})$ is zero at $\dot{\gamma}=0$, and increases monotonically with the increase of $\dot{\gamma}$. For a completely structured material initially at rest, if it is suddenly subjected to a constant shear rate (artificially assumed here), the structure breakdown rate will increase from 0 to a maximum value, after that it begins to decrease. However, if the function $f(\tau, \dot{\gamma})$ is dependent on shear rate only, $f(\tau, \dot{\gamma})$ does not change with time. As a consequence, the breakdown term $\lambda f(\tau, \dot{\gamma})$ is a maximum at the onset of the applied shear rate (because $\lambda=1$ and $f(\tau, \dot{\gamma})$ is constant), and decreases as $\lambda$ decreases. This is not consistent with the actual evolution of $\lambda$. Therefore, de Souza Mendes $(2009 ; 2011)$ came to the conclusion that it is non-physical to assume that the destruction of microstructure is a function of shear rate. One thing to note is that the nonphysical responses mentioned above lies in the viscoelastic part before the yield point. Therefore, it does not show up in the thixotropic models (e.g. visco-plastic models) without considering the viscoelastic part before the yield point.

To overcome this problem, the two models proposed by de Souza Mendes $(2009 ; 2011)$ assume that the breakdown term of the structural kinetic equation is a function of the instantaneous stress. However, for the thixotropic dominated regime (away from the yield point) when the network structure is broken down into isolated flocs suspended in the liquid, it is shown that the surface shear stress experienced by an isolated floc is proportional to the shear rate raised to a power (Barnes, 1997). Therefore, in this regime it is reasonable to assume that the breakdown term of the structural kinetic equation is dependent on shear rate. This is also the reason why most of the kinetic equations in the viscoplastic model assume that the breakdown term is dependent on shear rate.

To take consideration of the initial elastic dominated regime and the thixotropic (and/or viscous) dominated regime, here we assume that $f(\tau, \dot{\gamma})$ is a function of a combination of shear stress and shear rate, e.g. the rate of energy dissipation $\phi$, which is defined as $\phi=\tau \dot{\gamma}$ in the simple shear flow. For constant shear rate coditions, the shear stress will increase with time until it reaches its peak value, the energy dissipation rate $\phi$ will increase with time, and so do the values of $f(\tau, \dot{\gamma})$ and $\lambda f(\tau, \dot{\gamma})$, and the breakdown 
term $\lambda f(\tau, \dot{\gamma})$ will arrive at its maximum value near the peak shear stress point. This is in line with the actual evolution of $\lambda$.

Based on the above discussion, we propose the following kinetic equation to depict the evolution of the structural parameter $\lambda$ with time:

$$
\frac{\mathrm{d} \lambda}{\mathrm{d} t}=\frac{1}{1+\gamma^{n_{2}}}\left[a(1-\lambda)-b \lambda \phi^{m}\right]
$$

where $b$ is a kinetic constant for shear-induced breakdown and $m$ is a dimensionless constant.

In summary, the proposed model is composed of Eq. (2) and Eq. (4), and contains ten adjustable parameters, i.e. $\tau_{\mathrm{y}}, k$, $\Delta k, n_{1}, n_{2}, a, b, m, p, q$. The values of the model parameters can be obtained via a least-squares fitting to a transient flow curve, with the kinetic equation for the structural parameter $\lambda$ solved numerically by the fourth order Runge-Kutta discretization method.

\section{Materials and methods}

The crude oils used in this study were Daqing and Zhongyuan waxy crude, two representative waxy crude oils in China. In consideration of the thermal- and shearhistory dependence of rheological behavior of waxy crude, the oil specimens were pretreated to a temperature of $80{ }^{\circ} \mathrm{C}$ to resolve all wax crystals so as to remove the "memory" of the oil for better repeatability and comparability of the experimental data, and then placed at room temperature statically for $48 \mathrm{~h}$ before testing.

All measurements were performed with the coaxial cylinder sensor system (Z41Ti) of a stress-controlled rheometer (HAAKE MARS III), and the temperature of samples was controlled by a programmable water bath (AC 200) with temperature control accuracy of $0.01{ }^{\circ} \mathrm{C}$. A pretreated oil specimen was heated to $50{ }^{\circ} \mathrm{C}$ and held at that temperature for $20 \mathrm{~min}$. It was then loaded into the measuring cylinder preheated at $50{ }^{\circ} \mathrm{C}$ and held isothermally for $10 \mathrm{~min}$, and then statically cooled to the test temperature at a cooling rate of $0.5{ }^{\circ} \mathrm{C} / \mathrm{min}$. At test temperatures, the oil specimen was held isothermally for $45 \mathrm{~min}$ before testing to let the wax crystal structure fully develop. Thereafter, thixotropic measurements were performed isothermally. In this work, the chosen test temperatures for Daqing waxy crude were 32, 33,34 , and $35^{\circ} \mathrm{C}$; while for Zhongyuan waxy crude the test temperatures were 34,35 , and $36^{\circ} \mathrm{C}$. For each test a fresh specimen was used.

\section{Model validation}

In this section, the proposed model was validated respectively by stepwise shear rate tests and hysteresis loop tests. Furthermore, to check the validity and applicability of the model, we used the model parameters obtained from the stepwise shear rate test to predict the transient flow curve of hysteresis loop test.

\subsection{Stepwise shear rate test}

The stepwise shear rate test is the mostly used thixotropic test, since the coupled effects of time and shear rate/stress can be clearly separated in such experiments (Barnes, 1997). In this work, the stepwise shear rate test is adopted and the chosen shear rates are $1,2,4,8,16,32$, and $64 \mathrm{~s}^{-1}$. The experimental data and fitted results of the proposed model for Daqing waxy crude at $33^{\circ} \mathrm{C}$ and Zhongyuan waxy crude at $34{ }^{\circ} \mathrm{C}$ are shown in Figs. 1 and 2, respectively. As we are mostly interested in the viscoelastic regime at small shear strains and the post-yield region, the shear stress is plotted against the shear strain in the logarithmic scale and against the time in the linear scale. It can be seen in Figs. 1 and 2 that the fitted flow curve is very close to the measured flow curve. For the two waxy crudes, the average absolute deviations (AADs) between the fitted values and the measured data are within $2.0 \%$.

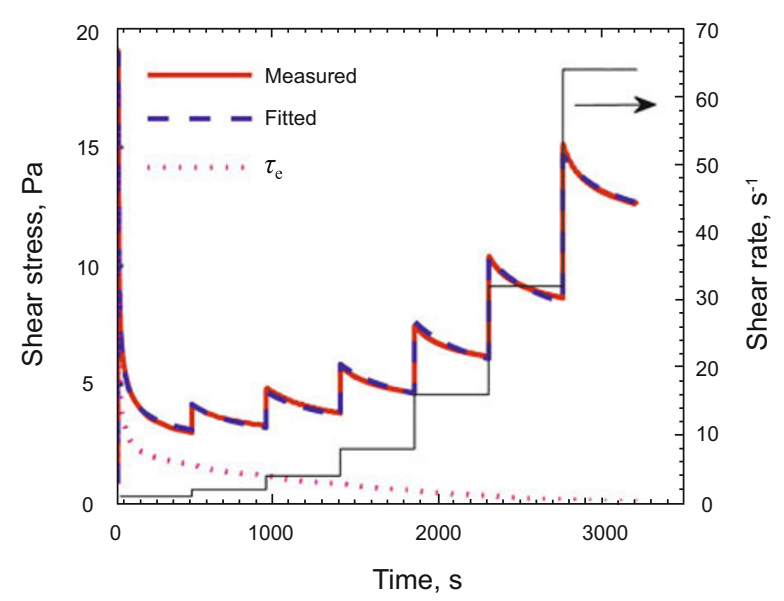

(a) Shear stress vs. shear time

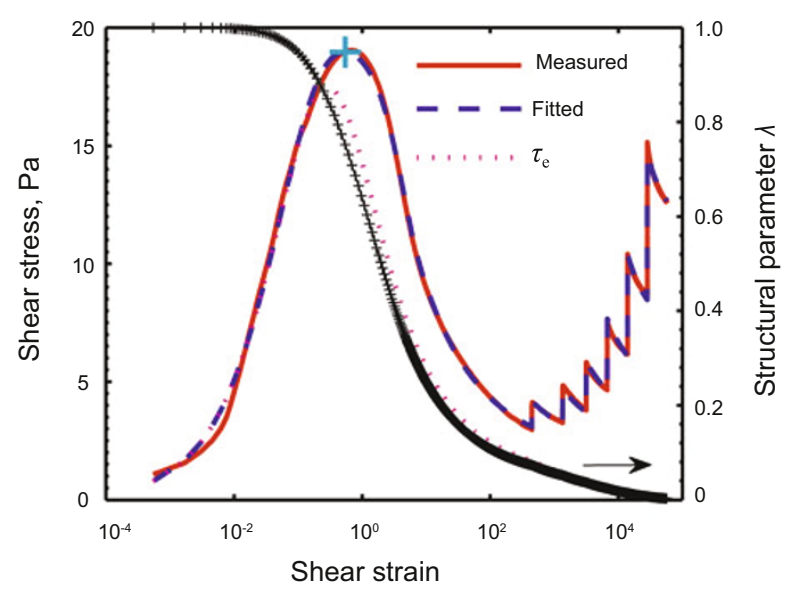

(b) Shear stress vs. shear strain

Fig. 1 Model fitting of stepwise shear rate test data for Daqing waxy crude at $33{ }^{\circ} \mathrm{C}$ (the cyan marker ' + ' in (b) corresponding to the peak shear stress point)

From Figs. 1(b) and 2(b), it can be observed that at the beginning of deformation, the mechanical response is mainly viscoelastic, and the shear stress increases with an increase in shear strain. The total shear stress predicted by the model is mainly made up of the elastic stress $\tau_{\mathrm{e}}$, with 


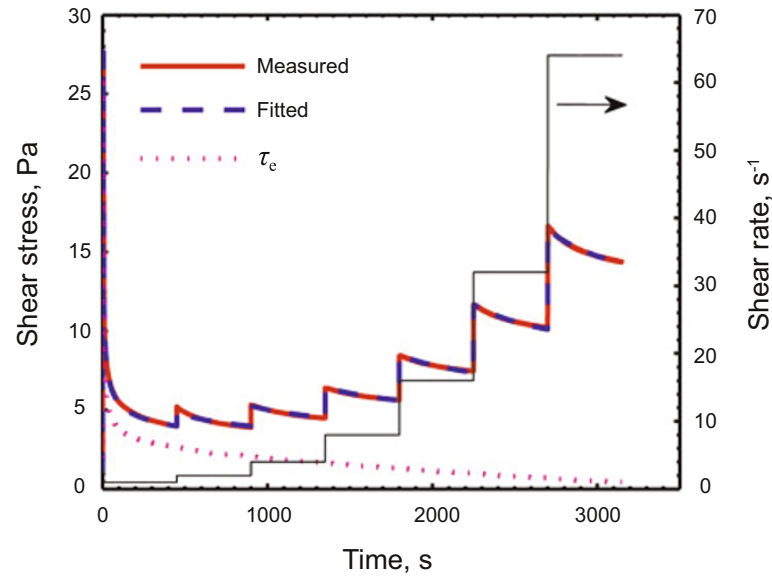

(a) Shear stress vs. shear time

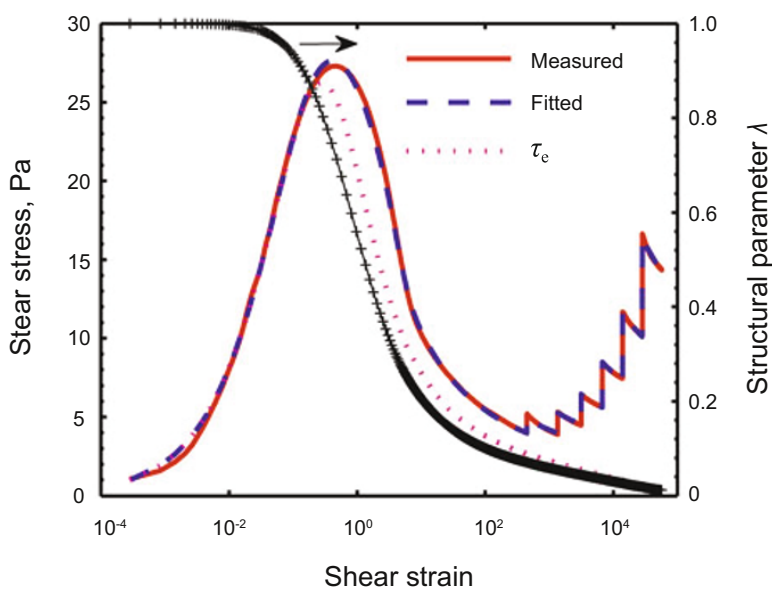

(b) Shear stress vs. shear strain

Fig. 2 Model fitting of stepwise shear rate test data for Zhongyuan waxy crude at $34{ }^{\circ} \mathrm{C}$

the viscous stress $\tau_{\mathrm{v}}$ very small. As the shear strain further increases, the structural parameter $\lambda$ begins to decrease and the mechanical response transfers from the viscoelastic regime to the viscoelastic-and-thixotropic coexistence regime, accompanying the yielding process. For the model, the predicted total shear stress changes from being dominated by $\tau_{\mathrm{e}}$ to being dominated by $\tau_{\mathrm{v}}$. While at higher shear rates, $\lambda$ is very small, and the predicted shear stress is mainly composed of $\tau_{\mathrm{v}}$, with $\tau_{\mathrm{e}}$ very small. In summary, the model predicts the transition from the "elastically" dominated region to the "viscously" dominated region nicely without a discontinuity and/or a deflection in the stress-strain curve.

For Daqing waxy crude at $33^{\circ} \mathrm{C}$, the detailed variation of structural parameter $\lambda$ with time is also shown in Fig. 3. Figs. 1 and 3 indicate that at low shear strains, the breakdown rate of $\lambda$ is very small and it increases as the shear strain increases. Near the yield point, the breakdown rate of $\lambda$ comes to its peak value, as can be observed in Fig. 1(b). After that, the structure buildup rate starts to increase while the structure breakdown rate decreases. As time goes on, the structure buildup and breakdown gradually reach dynamic equilibrium. Hence, the total shear stress gradually decays to its equilibrium value. For the following shear rates, when the shear rate is suddenly changed to another higher value, the shear stress also suddenly jumps to a higher stress owing to the jump of shear rate. The structure breakdown rate is also stepwise elevated, and then gradually decays as $\lambda$ gradually comes to its equilibrium value at each shear rate. The total shear stress at each shear rate also gradually decays to its equilibrium, as shown in Fig. 1.

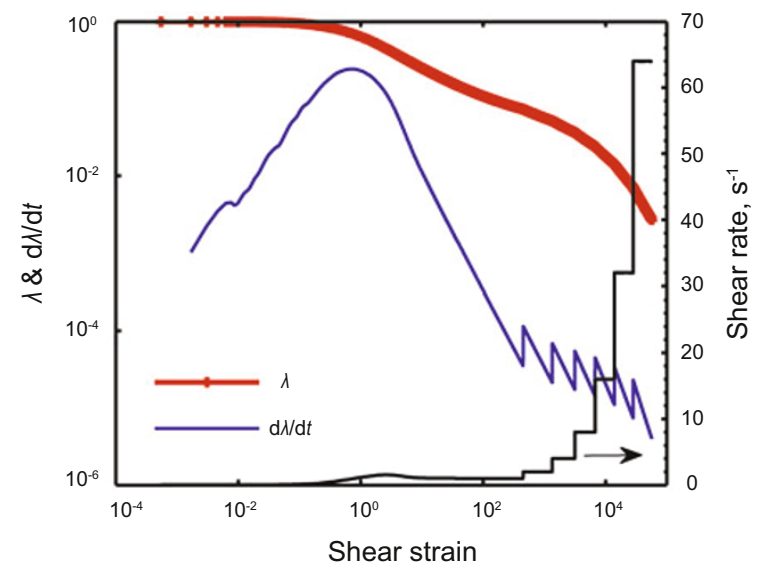

Fig. 3 Evolution of the structural parameter $\lambda$ in the stepwise shear rate test of Daqing waxy crude at $33{ }^{\circ} \mathrm{C}$

\subsection{Hysteresis loop test}

The hysteresis loop measurement is a common method for demonstrating the thixotropic behavior of complex fluids (Barnes, 1997; Labanda and Llorens, 2006). The hysteresis loop test in this study consists of two cycles of linear increase of shear rate from 0 to $50 \mathrm{~s}^{-1}$ in $200 \mathrm{~s}$ and then linear decrease to $0 \mathrm{~s}^{-1}$ in the same time period. Fig. 4 shows the experimental data and fitted results of the hysteresis loop test for Daqing waxy crude at $33{ }^{\circ} \mathrm{C}$. It can be observed that the fitted flow curve is nearly superposed with the experimental one for the total data range. The AADs are within 5.0\% for Daqing and Zhongyuan waxy crude at the test temperatures.

Like the stepwise shear rate test, the initial shear stress shows up a stress overshoot and undershoot, indicating the viscoelastic properties of waxy crude. Near the yield point, the elastic stress $\tau_{\mathrm{e}}$ decreases sharply to a very small value, and the total shear stress predicted by the model gradually changes from being dominated by the elastic stress $\tau_{\mathrm{e}}$ to being dominated by the viscous stress $\tau_{\mathrm{v}}$, as analyzed in the section 4.1.

To further estimate the proposed model, here we used the model parameters obtained from stepwise shear rate test to predict the transient flow curve of the hysteresis loop test. Fig. 5 shows the predicted results of Daqing waxy crude at $33{ }^{\circ} \mathrm{C}$ as an example. It can be seen that the thixotropic behavior of the hysteresis loop test can be described by the model on the whole. The AADs are within $18 \%$ for Daqing 


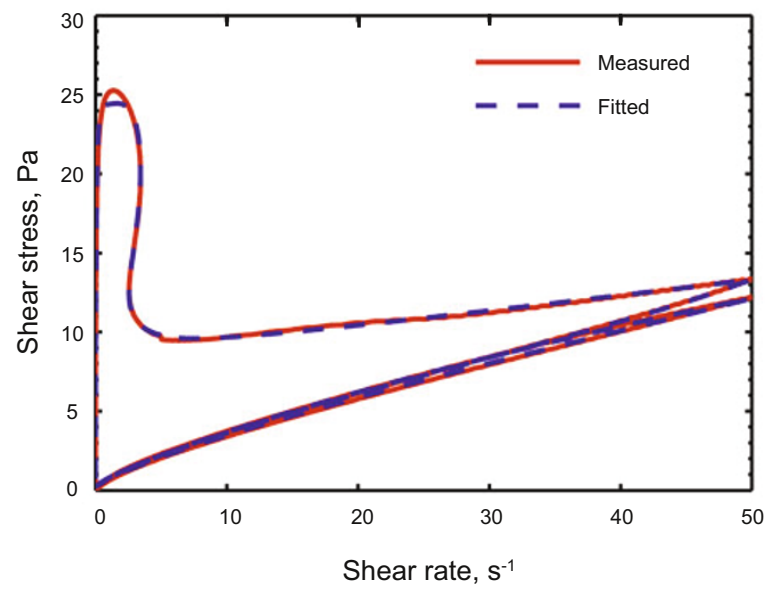

(a) Shear stress vs. shear rate

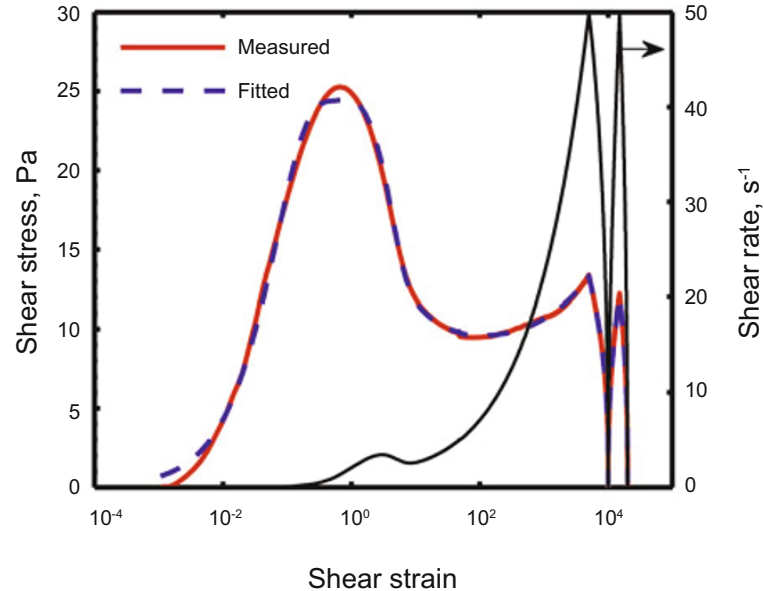

(b) Shear stress vs. shear strain

Fig. 4 Model fitting of hysteresis loop test data for Daqing waxy crude at $33^{\circ} \mathrm{C}$

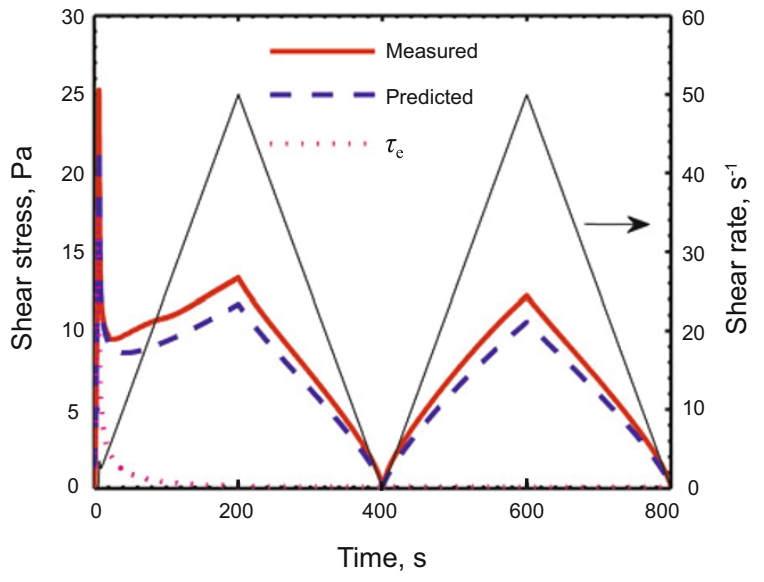

(a) Shear stress vs. time

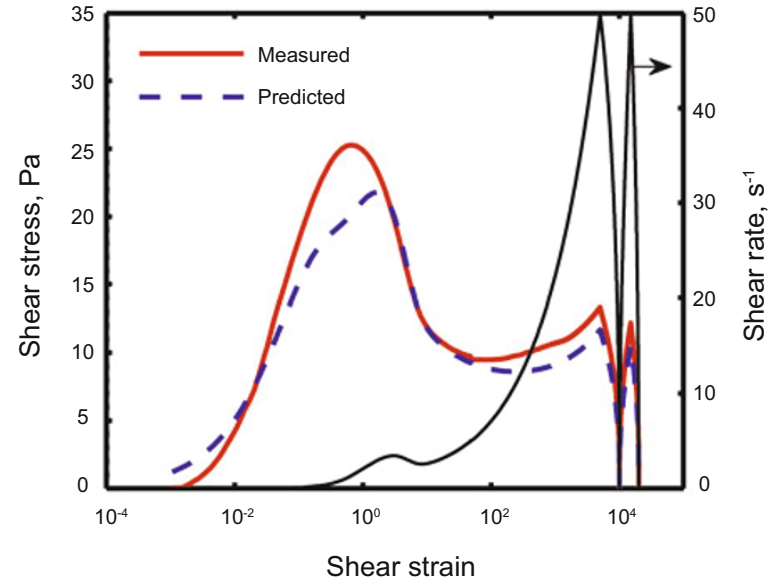

(b) Shear stress vs. shear strain

Fig. 5 Model predictions of the hysteresis loop for Daqing waxy crude at $33{ }^{\circ} \mathrm{C}$ (using model parameters from stepwise shear rate test)

and Zhongyuan waxy crude at the test temperatures. Near the peak shear stress point, the deviation between the fitted value and the measured data is relatively high. This is caused by the fast decay of the structural parameter $\lambda$.

\section{Conclusions}

To completely depict the viscoelasto-thixotropic behavior of waxy crude, a viscoelasto-plastic model with ten unknown parameters is proposed, based on the Mujumbar model and the Zhu model. The proposed model is validated by the data from stepwise shear rate tests and the hysteresis loop tests of Daqing and Zhongyuan waxy crude. The results showed that the average absolute deviations of the two measurements are within $2.0 \%$ and $5.0 \%$, respectively. The model depicts the whole rheological response before and after the yield point well without a discontinuity, from the initial elastic dominated region, through the viscoelasticity and thixotropy coexistence region accompanied with the yielding process, and finally to the viscous dominated region. Moreover, the model is further validated by predicting the transient flow curve of the hysteresis loop test with the model parameters obtained from the stepwise shear rate test, and the results show that the agreement between the predictions and measurements is satisfactory.

\section{Acknowledgements}

The authors are grateful for the financial support from the National Natural Science Foundation of China (Grant No. 51134006) and Science Foundation of China University of Petroleum (Beijing) (Grant No. LLYJ-2011-55).

\section{References}

Barnes H A. Thixotropy-A review. Journal of Non-Newtonian Fluid Mechanics. 1997. 70(1-2): 1-33

Chang C, Boger D V and Nguyen Q D. The yielding of waxy crude oils. Industrial \& Engineering Chemistry Research. 1998. 37(4): 15511559

Coussot P, Nguyen Q D, Huynh H T, et al. Viscosity bifurcation in thixotropic, yielding fluids. Journal of Rheology. 2002. 46(3): 573589

de Souza Mendes P R. Modeling the thixotropic behavior of structured 
fluids. Journal of Non-Newtonian Fluid Mechanics. 2009. 164(1-3): $66-75$

de Souza Mendes P R. Thixotropic elasto-viscoplastic model for structured fluids. Soft Matter. 2011. 7(6): 2471-2483

Doraiswamy D, Mujumdar A N, Tsao I, et al. The Cox-Merz rule extended: a rheological model for concentrated suspensions and other materials with a yield stress. Journal of Rheology. 1991. 35(4): 647-685

Dullaert K and Mewis J. A structural kinetics model for thixotropy. Journal of Non-Newtonian Fluid Mechanics. 2006. 139(1-2): 21-30

Fang B and Jiang T Q. A novel constitutive equation for viscoelasticthixotropic fluids and its application in the characterization of blood hysteresis loop. Chinese Journal of Chemical Engineering. 1998. 6(3): 264-270

Fang B, Song D Y and Jiang T Q. Study of the novel 7-parameter constitutive equation for viscoelastic-thixotropic fluids. The Sixth National Conference on Rheology, 1-3 November 1999, Wuhan, China. 242-244 (in Chinese)

Houska M. Engineering Aspects of the Rheology of Thixotropic Liquids. Ph.D. Thesis. Czech Technical University of Prague. 1981

Huang S X and $\mathrm{Lu} \mathrm{C}$. The characterization of the time-dependent nonlinear viscoelastic of an LDPE melt using a simple thixotropy model. Acta Mechanica Sinica. 2005. 21(4): 330-335

Jia B L and Zhang J J. A six-parameter thixotropic model for waxy crude oil. Acta Petrolei Sinica. 2012. 33(6): 1080-1085 (in Chinese)

Labanda J and Llorens J. A structural model for thixotropy of colloidal dispersions. Rheologica Acta. 2006. 45(3): 305-314

Liddel P V and Boger D V. Yield stress measurements with the vane. Journal of Non-Newtonian Fluid Mechanics. 1996. 63(2-3): 235-261

Livescu S. Mathematical modeling of thixotropic drilling mud and crude oil flow in wells and pipelines: a review. Journal of Petroleum Science and Engineering. 2012. 98-99: 174-184

Magda J, El-Gendy H, Oh K, et al. Time-dependent rheology of a model waxy crude oil with relevance to gelled pipeline restart. Energy and Fuels. 2009. 23(3): 1311-1315

Mewis J and Wagner N J. Thixotropy. Advances in Colloid and Interface Science. 2009. 147-148: 214-227
Mujumdar A, Beris A N and Metzner A B. Transient phenomena in thixotropic systems. Journal of Non-Newtonian Fluid Mechanics. 2002. 102(2): 157-178

Oh K and Deo M. Characteristics of wax gel formation in the presence of asphaltenes. Energy \& Fuels. 2008. 23(3): 1289-1293

Oh K, Jemmett M and Deo M. Yield behavior of gelled waxy oil: effect of stress application in creep ranges. Ind. Eng. Chem. Res. 2009. 48 (19): 8950-8953

Putz A M V and Burghelea T I. The solid-fluid transition in a yield stress shear thinning physical gel. Rheologica Acta. 2009. 48(6): 673-689

Rønningsen H P. Rheological behaviour of gelled, waxy North Sea crude oils. Journal of Petroleum Science and Engineering. 1992. 7(3-4): $177-213$

Teng H X and Zhang J J. A new visco-plastic thixotropic model for waxy crude. Proceedings of the eleventh National Conference on Rheology. 16-18 October 2012, Langfang, China. 138-141 (in Chinese)

Toorman E A. Modelling the thixotropic behaviour of dense cohesive sediment suspensions. Rheologica Acta. 1997. 36(1): 56-65

Visintin R F G, Lapasin R, Vignati E, et al. Rheological behavior and structural interpretation of waxy crude oil gels. Langmuir. 2005. 21(14): 6240-6249

Yziquel F, Carreau P J, Moan M, et al. Rheological modeling of concentrated colloidal suspensions. Journal of Non-Newtonian Fluid Mechanics. 1999. 86(1-2): 133-155

Zhang J J, Guo L P and Teng H X. Evaluation of thixotropic models for waxy crude oils based on shear stress decay at constant shear rates. Applied Rheology. 2010. 20(5): 53944-53950

Zhang J J and Liu X. Some advances in crude oil rheology and its application. Journal of Central South University of Technology. 2008. 15: 288-292

Zhu C and Smay J E. Thixotropic rheology of concentrated alumina colloidal gels for solid freeform fabrication. Journal of Rheology. 2011. 55(3): 655-672

Zhu Y R, Zhang J J, Li H Y, et al. Characteristic temperatures of waxy crude oils. Petroleum Science. 2007. 4(3): 57-62

(Edited by Sun Yanhua) 